\title{
Structural biological study of self-resistance determinants in antibiotic-producing actinomycetes
}

\author{
Masanori Sugiyama \\ As antibiotics act to inhibit the growth of bacteria, the drugs are useful for treating bacterial infectious diseases. However, \\ microorganisms that produce antibiotics must be protected from the lethal effect of their own antibiotic product. In this review, \\ the fruit of our group's current research on self-protection mechanisms of Streptomyces producing antibiotics that inhibit DNA, \\ protein and bacterial cell wall syntheses will be described.
}

The Journal of Antibiotics (2015) 68, 543-550; doi:10.1038/ja.2015.32; published online 15 April 2015

\section{INTRODUCTION}

Actinomycetes, which are mainly found in soil, are industrially important, because they have provided major practical antibiotics and useful enzymes. ${ }^{1}$ Streptomyces is a genus of actinomycetes that comprises more than $60 \%$ of antibiotics discovered until now. The genus Streptomyces is a Gram-positive filamentous bacterium that undergoes a complex morphological differentiation, resembling that of filamentous fungi.

Judging from the fact that the arrival of antibiotics remarkably reduced the death rate by infectious diseases, infectious disease had seemed to be overcome. However, as a result of the disorderly use of antibiotics for the treatment of infectious diseases, bacteria tolerance to the drugs has certainly appeared. ${ }^{2-4}$ Now, the warlike circle between the development of new antibiotics and the appearance of drugresistant bacteria goes on.

Pathogenic bacteria, which are resistant to aminoglycoside antibiotics such as streptomycin (Sm), neomycin and kanamycin, harbor enzymes that add phosphate, acetyl or adenyl group to the antibiotic. ${ }^{2}$ Each enzyme modifies the antibiotic as a way of protecting itself from the lethal effects of the antibiotic. A system is also known for modifying the normally sensitive target of the antibiotic and rendering it insensitive. As an example, erythromycin-resistant bacteria ${ }^{5}$ harbor an enzyme that attaches a methyl group at a specific point on the ribosomal RNA, rendering the ribosome insensitive to inhibition. ${ }^{6} \mathrm{~A}$ third way, which is exemplified by tetracycline-resistant pathogenic bacteria, ${ }^{5}$ depends on a transmembrane protein channel that pumps the antibiotic out of the cells by coupling antibiotic excretion with proton import. ${ }^{7}$

Antibiotic-producing microorganisms must be protected from lethal effect of their own product. The ability inherent in the producer organisms is called self-resistance. It is very important and significant in developing therapeutic drugs to infectious diseases to know similarity or antithesis between drug resistance mechanism of pathogenic bacteria and self-resistance mechanism of antibioticproducing microorganisms.

In general, antibiotics are produced during the late exponential and late stationary growth of the producer organism and secreted into the medium, but not during the early stage of growth. Recent research has shown that antibiotic-producing microorganisms have a system that leads to transcription of the antibiotic-biosynthesizing genes at the appropriate time. To act effectively for self-resistance to its own antibiotic product, antibiotic-modifying and target-protecting enzymes, or the antibiotic excretion system must be present in the producer organisms, when the biosynthetic pathway begins to produce the antibiotic. ${ }^{8}$

Antibiotics can be classified into several groups by the differences in the action mechanism and chemical structure. In this study, the fruit of our group's research on the self-protection mechanisms of Streptomyces producing inhibitors of the DNA, protein and bacterial cell wall syntheses will be mainly described.

\section{ANTIBIOTICS THAT INHIBIT DNA SYNTHESIS}

Bleomycin $(\mathrm{Bm})$, which inhibits the growth of bacterial and tumor cells, is an antibiotic consisting of sugar and peptide, and has molecular weight of $\sim 1500 .{ }^{9} \mathrm{Bm}$, which is used as a potent anticancer drug due to its DNA-cleaving ability, requires molecular oxygen and Bm-bound ferrous ion. ${ }^{9}$ The Bm family of antibiotics, produced by Streptomyces verticillus, is secreted as a $\mathrm{Cu}$ (II)-bound form into a culture broth. As shown in Figure 1, bleomycin $\mathrm{A}_{2}\left(\mathrm{Bm} \mathrm{A}_{2}\right)$, a member of the $\mathrm{Bm}$ family with a $\gamma$-aminopropyldimethylsulphonium moiety, is clinically used for non-Hodgkin lymphomas, squamous cell carcinomas and testicular tumors. ${ }^{10,11}$

The cleavage of DNA is thought to account for the antineoplastic activity of $\mathrm{Bm} \mathrm{A}_{2}$, which proceeds via either the generation of the 
oxoiron $\mathrm{Bm}$ complex by exchanging $\mathrm{Cu}(\mathrm{II})$ for $\mathrm{Fe}(\mathrm{II})$ or the oxygenation of the $\mathrm{Cu}(\mathrm{I})$-bound $\mathrm{BmA}_{2}$ complex reduced from $\mathrm{Cu}$ (II)-bound $\mathrm{BmA}_{2}$ by the thiol groups. ${ }^{12,13}$

The author has tried to investigate the self-protection mechanism of Bm-producing S. verticillus ATCC15003 with Professor Julian Davies of the Institut Pasteur, Paris, and to clone the self-resistance determinant-encoding genes. ${ }^{14}$ First, to ascertain whether the microorganism harbors an enzyme that loses the antibacterial activity of Bm, the cell-free extract prepared from the mycelium of $S$. verticillus was incubated with $\mathrm{Bm}$. Interestingly, the antibiotic activity of $\mathrm{Bm}$ completely disappeared with the addition of acetyl coenzyme A to the incubation mixture, demonstrating that the $\mathrm{Bm}$ producer has an enzyme that acetylates $\mathrm{Bm} .{ }^{14}$ Using enzymatically prepared acetyl-Bm $\mathrm{A}_{2}$, the site of acylation was determined by nuclear magnetic resonance analysis ${ }^{15}$; the acetylation site was the primary amino acid group of the $\beta$-aminoalanine moiety of $\mathrm{Bm}$, leading us to conclude that the enzyme is $\mathrm{Bm} \mathrm{N}$-acetyltransferase (BAT). Acetyl- $\mathrm{Bm} \mathrm{A} \mathrm{A}_{2}$ had no detectable antibacterial activity and did not induce in vitro DNA degradation.

Furthermore, a gene encoding BAT was successfully obtained as a 6kb DNA fragment from the $S$. verticillus ATCC15003 chromosome. ${ }^{14}$ In order to localize the BAT gene on the DNA fragment, the fragment was digested with suitable restriction enzymes, suggesting that there is also another Bm-resistance gene beside the gene encoding BAT. The gene that consists of a 366-bp open reading frame (ORF) starts with GTG $(G=+1)$ and ends at the stop codon TGA $\left(T^{367}\right)$; this ORF encodes an acidic protein that consists of 122 amino acids. The protein, referred to as BLMA, was confirmed to have a strong affinity for $\mathrm{Bm}$. The genes for BLMA and BAT with molecular weight of $\sim 13,000$ and 34,500, respectively, were named $b \operatorname{lm} A$ and $b \operatorname{lm} B$, respectively. Interestingly, tallysomycin-producing Streptoalloteichus hindustanus $^{16}$ also carries a Bm-resistance determinant, the Shble protein. The similarity of amino acid sequences between BLMA and the Shble protein was $60 \%$.

BLMA, which was obtained by an Escherichia coli host-vector system and purified to homogeneity, was crystallized to determine the tertiary structure by X-ray crystallographic analysis. ${ }^{17,18}$ The BLMA structure, determined by the single isomorphous replacement method, including the anomalous scattering effect, was refined at a $1.5 \AA$ resolution (Figure 2). The final R-factor was $19.0 \%$ and $\mathrm{R}$ (free) was $22.1 \%$, including 91 water molecules. The crystal packing showed a dimer form, which was generated by arm exchange. The $1.5-\AA$ high resolution allowed an analysis of the side-chain disorder of BLMA. The structural comparison between BLMA and the Shble protein showed that a Ser100-Gly103 loop was farther from the groove, which

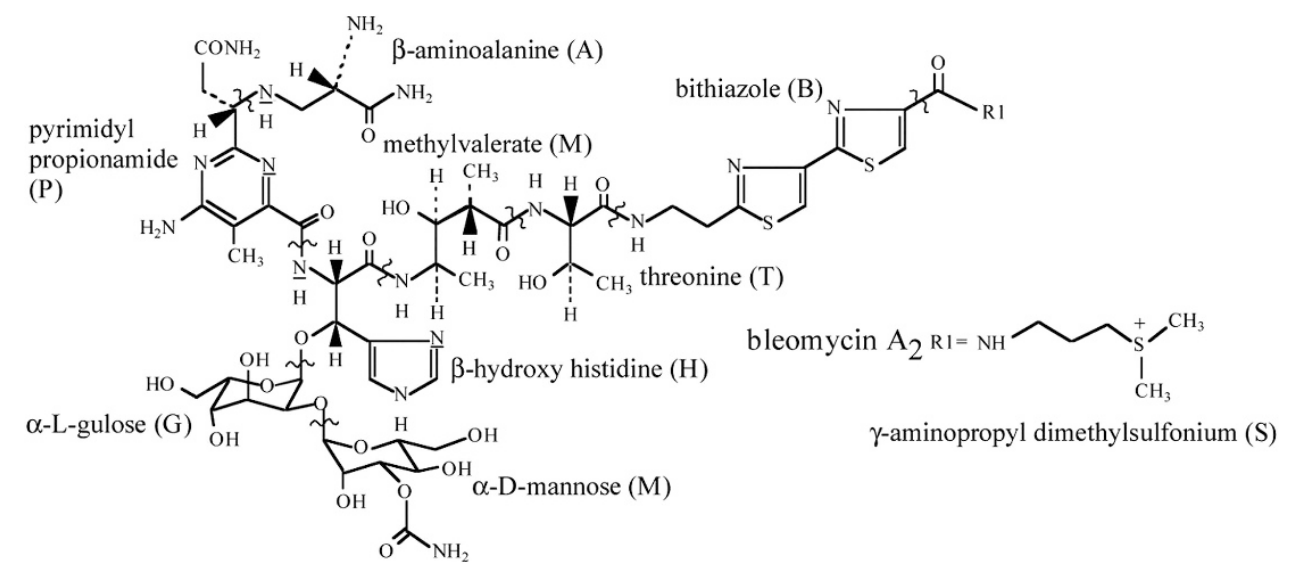

Figure 1 The structure of $\mathrm{Bm} \mathrm{A}_{2}$. Wavy lines indicate the junction between the molecular units comprising $\mathrm{Bm} \mathrm{A}_{2}$. The underlined $\mathrm{N}$ atoms are the putative equatorial ligands to the metal ion and the acetylation site of Bm by BAT is indicated by a circle.
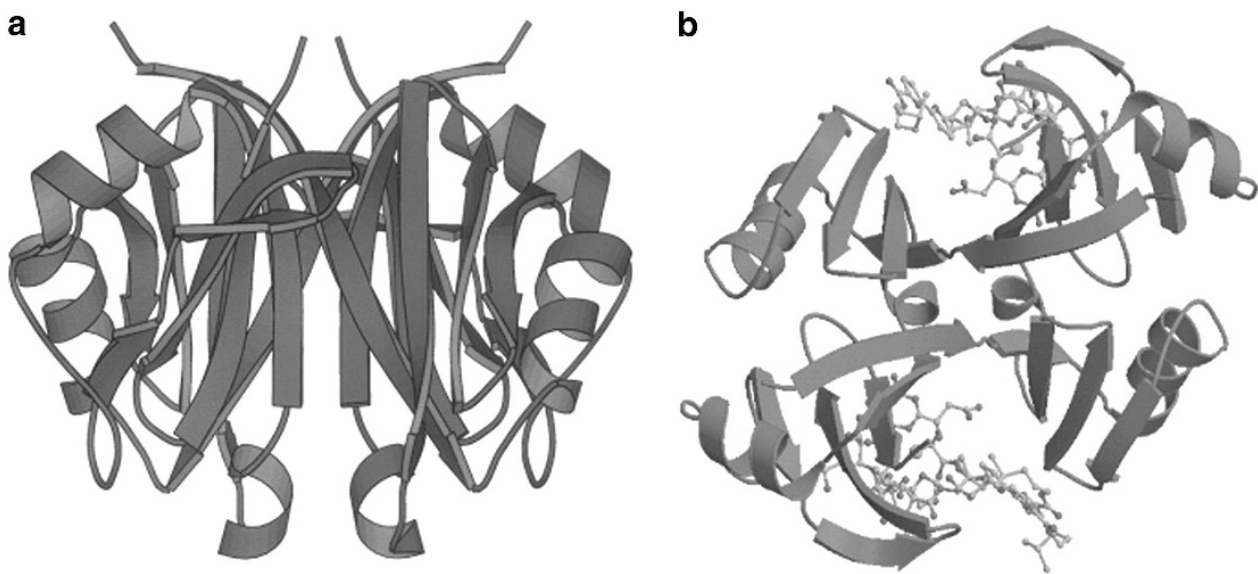

Figure 2 The tertiary structure of BLMA. (a and $\mathbf{b}$ ) Structures judging from the top and the side, respectively. (b) Two Bm molecules bind to the dimeric BLMA molecule. A full color version of this figure is available at The Journal of Antibiotics journal online. 
is a Bm-binding site, in BLMA than in the Shble protein. ${ }^{19}$ Furthermore, the hydrophobicity of the groove in BLMA is much lower than that in the Shble protein. The structural differences between both proteins may be responsible for the observation that a half-saturating concentration of Bm is higher for BLMA than for the Shble protein. ${ }^{14}$

$\mathrm{Bm}$ in the culture broth of $S$. verticillus is complexed with $\mathrm{Cu}^{2+}[\mathrm{Cu}$ (II)]. Our group determined the X-ray crystal structures of the $\mathrm{Cu}(\mathrm{II})$ bound and metal-free types of $\mathrm{Bm}$ at a high resolution of 1.6 and $1.8 \AA$, respectively, which are complexed with BLMA. ${ }^{20}$

Transposon Tn5 carries a gene that confers resistance to $\mathrm{Bm}$ in E. coli..$^{21-23}$ Our group has crystallized the Bm-resistance determinant, designated BLMT, ${ }^{24}$ and determined the X-ray crystal structures of BLMT uncomplexed and complexed with $\mathrm{Bm}$ at 1.7 and $2.5 \AA$ resolutions, respectively. ${ }^{25}$ BLMT takes a dimer structure with two Bm-binding pockets composed of two large concavities and two long grooves. The amino acid sequence homology between BLMA and the Shble protein is $60 \%$, whereas that between BLMT and BLMA is only $20 \%-25 \%$. Nevertheless, the superposition of the main-chain atoms of BLMT on that of BLMA shows that the overall structure of BLMT is almost the same as that of BLMA (Figure 3). This crystal structure of BLMT complexed with $\mathrm{Bm}$ gives a precise mode for binding the antibiotic to BLMT. The conformational change of BLMT generated by binding to Bm occurs at a $\beta$-turn composed of the residues from Gln97 to Thr102.

Crystallographic analysis of Bm bound to BLMT shows that two thiazolium rings of the bithiazole moiety are in the trans conformation. The axial ligand, which binds a metal ion, seems to be the primary amine in the $\beta$-aminoalanine moiety. This has been the first report to determine the X-ray crystal structure of $\mathrm{Bm}$, showing that the bithiazole moiety of $\mathrm{Bm}$ is far from the metal-binding domain, that is, Bm complexed with BLMT takes a more extended form than does the drug complexed with DNA.

Thus, we have shown by the structural studies that BLMA, BLMT and the Shble protein have commonly two Bm-binding pockets formed by the alternate arm exchange of two monomeric molecules.

Almost all strains of methicillin-resistant Staphylococcus aureus isolated at Hiroshima University Hospital, Japan, from October 1990 to April 1992, were resistant to Bm and kanamycin, although Bm was never used as an antibacterial agent. From our observation that the Bm-resistance properties did not disappear, even when the methicillin-

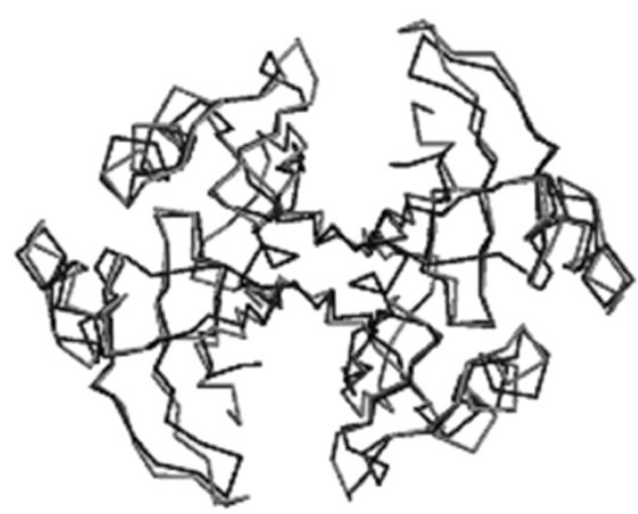

Figure 3 The superposition of the main-chain atoms of BLMT on that of BLMA. This figure shows that the overall structure of BLMT is almost the same as that of BLMA. Black and gray lines are BLMA and BLMT, respectively. resistant $S$. aureus cells were treated with ethidium bromide as a plasmid-curing agent, ${ }^{26}$ we believed that these strains were resistant to the drug as a result of the gene's presence in the chromosome rather than in the plasmid.

Our group has successfully cloned a DNA fragment containing a gene that confers $\mathrm{Bm}$ resistance from the chromosomal DNA of methicillin-resistant $S$. aureus strain B-26 isolated at Hiroshima University Hospital, showing that a Bm-resistance gene derived from the B-26 chromosome, designated blmS, is identical to that from staphylococcal plasmid pUB110. ${ }^{27,28}$ The nucleotide sequence of the 5.1-kb HindIII fragment containing the $\mathrm{Bm}$ - and kanamycin -resistance gene showed that the DNA sequence contains the duplicated target sequence (GATTAGAT) consisting of $8 \mathrm{bp}$ for transposase and the entire nucleotide sequence of plasmid pUB110, together with the sequence of inverted repeats (16 bp), designated IR-r and IR-1 in IS431mec. The 8-bp duplication sequence, produced by the transposable element, was first found by us, suggesting that the Bm resistance in methicillin-resistant $S$. aureus B-26 can be attributed to the IS431mec-mediated integration of pUB110 into the chromosome. ${ }^{29}$

We have also showed that both BLMA and BLMS quench antibacterial activity. To imnuno characterize the Bm-binding protein, we have constructed a monoclonal antibody against BLMA. The antibody did not cross-react to BLMS or the Shble protein. Although the ability of Bm to cleave DNA was eliminated by binding BLMA to $\mathrm{Bm}$, the Bm-induced DNA degradation was restored by preincubating BLMA with the anti-BLMA monoclonal antibody. ${ }^{30}$

Our group has also crystallized BAT under both a terrestrial and a microgravity environment in the International Space Station, in collaboration with JAXA (Japan Aerospace Exploration Agency). In addition to substrate-free BAT, the crystal structures of BAT in a binary complex with CoA and in a ternary complex with $\mathrm{Bm}$ and CoA were determined. ${ }^{31}$ The tertiary structure of a BAT monomer is shown in Figure 4a. The enzyme takes a dimer form for catalysis. In the orthorhombic crystal, only one CoA molecule for the BAT dimer was identified for the BAT dimer, whereas two CoA molecules were observed in the trigonal crystal. CoA binds to the narrow entrance of a tunnel in the N-terminal domain, with its thiol group reaching the midpoint of the tunnel. The nucleotide portion of CoA is extended outside the tunnel entrance and largely exposed to the bulk solvent. An examination of the electrostatic potential revealed a particularly basic region near the pyrophosphate and adenosine $3^{\prime}$-phosphate groups in CoA. Mercaptoethylamine, the pantothenate moieties and the pyrophosphate group in CoA react with BAT by forming a large number of hydrogen bonds and van der Waals contacts. A linear part of CoA binds along the $\beta 5$ strand and wedges between this strand and the $\beta 6$ strand. The pantetheine moiety forms hydrogen bonds with the main-chain atoms of Trp97 and Leu99. In addition, the side chains of Val40, Trp43, Trp44, Trp97, Leu98, Leu99, Trp104 and Ile133 contribute to the formation of a hydrophobic pocket suitable for accommodating the pantetheine moiety. The pyrophosphate group in CoA forms hydrogen bonds with the backbone amide groups of Gly105, His106, Gly107, Ala109 and Thr110, and with the side chains of Thr110, Arg139 and Arg146. The adenosine moiety in the CoA molecule is weakly defined in the electron density.

In the ternary complex structure obtained by co-crystallization with $\mathrm{CoA}$ and $\mathrm{Bm} \mathrm{A}_{2}$, one $\mathrm{CoA}$ and two $\mathrm{Bm} \mathrm{A}_{2}$ molecules were observed to bind to a BAT dimer (Figure $4 \mathrm{~b}$ ). A Bm-binding site in BAT is extended from an $\mathrm{N}$-terminal domain of one monomer to the dimer interface formed by the two C-terminal domains. In detail, a DNAbinding domain of $\mathrm{Bm}$ is accommodated in a canyon-like groove formed by the two C-terminal domains of BAT, where a bithiazole 


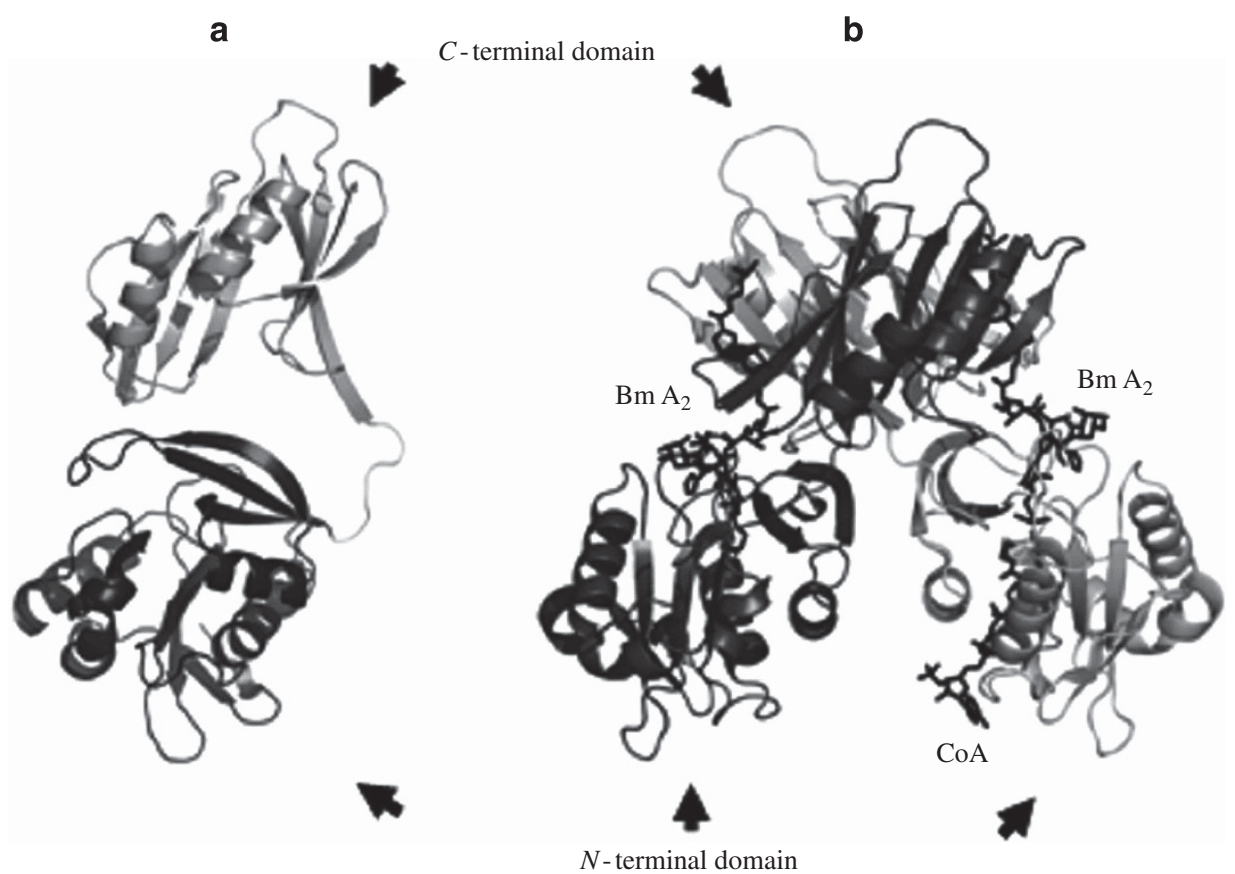

Figure 4 Overall structure of BAT. Ribbon views of a monomeric (a) and a dimeric (b) form of BAT in the crystal structure of a ternary complex bound with two $\mathrm{Bm} \mathrm{A}_{2}$ molecules and one $\mathrm{CoA}$ molecule.

moiety of $\mathrm{Bm}$ is sandwiched. At one side of the bithiazole-binding groove, an indole ring of Trp280 from one monomer is positioned, whereas two benzene rings of Phe211 and Phe219 from the other monomer are present on the other side. Metal binding and sugar domains of $\mathrm{Bm}$ are bound to a funnel-like surface of the $\mathrm{N}$-terminal domain of BAT. The pyrimidinylpropionamide and $\beta$-aminoalanine moieties of Bm enter deeply into the tunnel by forming a large number of van der Waals contacts with side chains of Trp44, Thr45, Leu87, Thr90, Trp132 and Tyr159, and hydrogen bonds with the side chains of Thr96 and Gln157. The positional relationship between the metal-binding and DNA-binding domains is not consistent between two $\mathrm{Bm}$ molecules bound to BAT, indicating that the Bm molecules adopt a conformation that reflects the different properties of the respective Bm-binding pockets. This is due to the fact that the connection between the bithiazole ring and the threonine moiety of $\mathrm{Bm}$ is flexible.

Mitomycin C (MMC), an inhibitor of DNA synthesis, is industrially produced by Sporobolus caespitosus. This antibiotic is a naturally occurring compound consisting of a pyrrolo (1, 2-a) indole ring system with an aziridine ring. Before interacting with DNA, MMC requires enzymatic activation by a one-electron pathway to a semiquinone or by a two-electron reduction pathway to a hydroquinone. The antibiotic is selectively toxic to chronically hypoxic carcinoma. The author's group was interested in investigating the selfprotection mechanism of MMC-producing microorganisms. We have used S. caespitosus as an MMC producer. ${ }^{32}$ It has been reported that Streptomyces lavendulae, another MMC-producing organism, expresses an MMC-binding protein with a molecular mass of $\sim 14 \mathrm{kDa}$, designated MRDP, encoded by a gene designated $m r d .^{33,34}$ As a consequence of the binding affinity exemplified by a dissociation constant in the micromolar range, MRDP has been determined to be important for the self-resistance of the producing organism.

Interestingly, the tertiary structure of $\mathrm{MRDP}^{35}$ using X-ray analysis exhibited a folding pattern similar to that observed in the crystal structure of BLMA, as well as other previously reported proteins of the glyoxalase/Bm-binding protein/dioxygenase superfamily, although no extensive sequence homology exists. This family of proteins includes proteins such as BLMT, a Bm-resistant protein from the E. coli transposon $\operatorname{Tn} 5,{ }^{25}$ a fosfomycin-resistant protein of Pseudomonas aeruginos $a^{36}$ and the glyoxalase I enzyme of $E$. coli. ${ }^{37}$ These proteins reveal a homodimer that consists of two $\beta \alpha \beta \beta \beta$ motifs from the $\mathrm{N}$-terminus and the $\mathrm{C}$-terminus in each monomer. Considering the architectural resemblance of the proteins, these proteins must have evolved from a common precursor.

In one approach to this speculation, we have cloned and characterized the mrd-like gene, which is a self-resistance determinant of S. caespitosus. We have demonstrated that the protein exhibited $100 \%$ sequence identity with that of $S$. lavendulae. Further, it was found that E. coli that carries mrd displays a high level of resistance to MMC and $\mathrm{BmA}_{2}$. Based on this observation, we have hypothesized that MRDP may display a defensive role against $\mathrm{BmA}_{2}$-induced DNA breakage. To clarify this, we have carried out a biochemical binding assay between $\mathrm{BmA}_{2}$ and MRDP, and determined the high-resolution crystal structure of MRDP in a complex with the $\mathrm{Cu}(\mathrm{II})$-bound form of $\mathrm{BmA}_{2}$ (Figure $\left.5 \mathrm{a}\right) .^{32}$ Figure $5 \mathrm{~b}$ shows the crystal structure of MRDP complexed with MMC.

The crystal structure of MDRP in complex with $\mathrm{Cu}$ (II)-bound $\mathrm{BmA}_{2}$, refined at a $1.4 \AA$ resolution, contains a homodimer with one molecule of the drug. The homodimer has a global fold almost identical to that of the apo form of MDRP and its MMC-bound structure, namely, a core of two twist-curl, eight-stranded $\beta$-sheets surrounded by four $\alpha$-helices. The dimer's interface is formed by the $\mathrm{N}$-terminal $\beta$-strand and a part of the hinge loop with the following $\beta$-strand.

\section{ANTIBIOTICS THAT INHIBIT PROTEIN SYNTHESIS}

Sm, which was discovered by Dr Selman A. Waksman, is well known as a specific remedy for tuberculosis, which occurs with infection by 
a

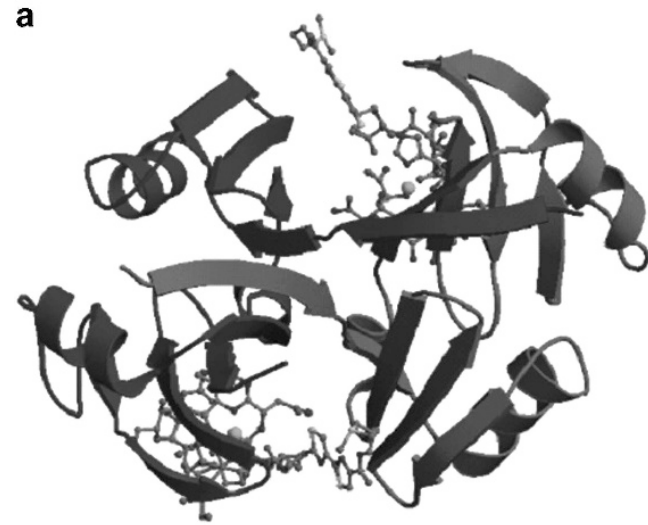

b

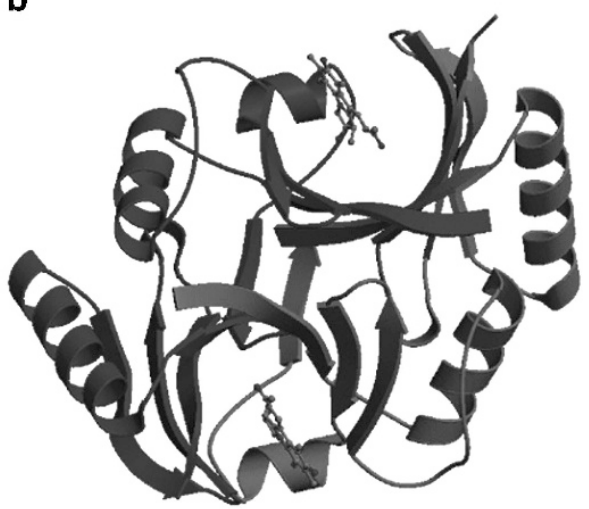

Figure 5 The tertiary structure of MRDP in a complex with $\mathrm{Cu}(\mathrm{II})$-bound $\mathrm{Bm} \mathrm{A}_{2}$. A full color version of this figure is available at The Journal of Antibiotics journal online.

Mycobacterium tuberculosis. Although the infectious disease had once decreased with the appearance of $\mathrm{Sm}$ in infectious therapy, the number of patients has recently begun to increase. The increase in morbidity is likely due to the decline in immunity caused by changes in the environment and diet. The occurrence of multi-drug-resistant M. tuberculosis is another cause of the return of tuberculosis. ${ }^{38}$

$\mathrm{Sm}$ inhibits protein synthesis by binding to the ribosomes of Smsensitive bacteria. As Sm-producing microorganisms must be protected from the lethal effect of $\mathrm{Sm}$, it is of interest to know whether the protein synthesis of the Sm-producing microorganism is inhibited by its own product. One research group could not demonstrate Sm's effect on protein synthesis in an in vitro system prepared from Streptomyces griseus, because the cell extract contained some factor that inhibited protein synthesis, using polyuridylate as mRNA. Another group reported that the binding affinity of $\left[{ }^{3} \mathrm{H}\right]$-labeled dihydro-SM with ribosomes from $S$. griseus is very low. ${ }^{8,39}$

As S. griseus is also known to produce protease, three kinds of protease inhibitors, magnesium titriplex, phenylmethylsulfonylfluoride and diisopropyl fluorophosphate, were used to prepare an in vitro protein-synthesizing system on Sm-producing S. griseus. Using these protease inhibitors, the author has successfully established an active in vitro protein synthesizing system consisting of 705 ribosomes and S-50 fractions (supernatant fluid fractionated by centrifuging the cellfree extract at $150000 \mathrm{~g}$ ) from $\mathrm{S}$. griseus cells, leading to the conclusion that the factor that inhibits the protein-synthesizing system of S. griseus is some protease produced by the cell itself. ${ }^{40}$ It has been demonstrated that $\mathrm{Sm}$ inhibits polyuridylate-dependent polyphenylalanine synthesis using the active in vitro system from the logarithmic phase cell of the Sm-producing S. griseus. The 70S ribosomes, prepared using protease inhibitors, were also examined for Sm-binding affinity; that is, the $70 \mathrm{~S}$ ribosomes were dissociated into $30 \mathrm{~S}$ and $50 \mathrm{~S}$ subunits by dialysis against a buffer that contained a decreased amount of magnesium acetate $(0.3 \mathrm{~mm})$ and then followed by incubation with $\left[{ }^{3} \mathrm{H}\right]$ dihydro-Sm before application to a sucrose density gradient centrifugation. As a result, after the fractionation, the peak of $\left[{ }^{3} \mathrm{H}\right]$ dihydro-Sm and that of $30 \mathrm{~S}$ ribosomal subunits appeared on the same fraction. This indicates that $\left[{ }^{3} \mathrm{H}\right]$ dihydro-Sm is bound to the $30 \mathrm{~S}$ ribosomal subunits of the ribosomes with protein-synthesizing activity. As $\mathrm{Sm}$ is mainly produced in the late stages of culture, the susceptibility of protein synthesis to Sm in the stationary phase of cell growth was also investigated. Polyphenylalanine synthesis was susceptible to $\mathrm{Sm}$, although the inhibitory rate was decreased. From these results, it can be concluded that the ribosomes of S. griseus are significantly susceptible to Sm. Consequently, S. griseus must have an Sm-resistant mechanism other than ribosomal resistance.

$\mathrm{Sm}$ is a basic water-soluble oligosaccharide (aminoglycoside) compound consisting of streptidine, L-storeptose and $\mathrm{N}$-methyl-Lglucosamine. In the biosynthesis of the streptidine moiety from myoinositol, the two nitrogen atoms on the cyclitol ring are introduced by transamination of inosose derivative. This reaction preferentially uses L-glucosamine as an amino donor. The transaminase also catalyzes an exchange reaction between syllo-inosamine and syllo-inosose. After syllo-inosamine is converted into syllo-inosamine phosphate with $O$-phosphotransferase, the first guanidine group is introduced by a transamidination reaction with arginine as the amidine donor. The transamidination reaction completes the synthesis of the second guanidine group of streptidine. Finally, the streptidine 6-phosphate formed is transferred to dTDP-dihydrostoreptose to form $O$ - $\alpha$-L-dihydrostreptose $(1 \rightarrow 4)$ streptidine 6-phosphate. The $\mathrm{N}$-methyl-L-glucosamine synthesized from D-glucose is coupled with $O$ - $\alpha$-L-dihydrostreptose $(1 \rightarrow 4)$ streptidine 6 -phosphate inside the cell. The resulting substance, dihydro-Sm 6-phosphate, was not found in the culture broth of $S$. griseus, suggesting that dihydro-Sm 6-phosphate is converted to Sm 6-phosphate in the cytoplasmic membrane. ${ }^{8}$ This Sm 6-phosphate as the final product in the Sm biosynthetic pathway is dephosphorylated by a periplasmic alkaline phosphatase and then secreted into the culture medium in an active form, Sm. Thus, $\mathrm{Sm}$ is produced as an inactive form in the S. griseus cell.

Our research group has shown that $\mathrm{Sm}$ bound to the $30 \mathrm{~S}$ ribosomal subunit of the Sm producer is effectively liberated by Sm 6-phosphotransferase in the presence of ATP. To clarify the roles of Sm 6-phosphotransferase, the activity in the S-150 fractions prepared from Sm-producing $S$. griseus was measured. The enzymatic activity was detected during all stages, whereas the activity in the S150 fraction from the stationary phase cell was obviously higher than that from the exponential phase cell. Although the binding affinity of $\mathrm{Sm}\left(\left[{ }^{3} \mathrm{H}\right]\right.$ dihydro-Sm) to the ribosomes and the permeability of Sm into S. griseus cells were significantly decreased during growth, we have concluded that the major contributor to the self-resistance of the producer organism is Sm 6-phosphotransferase. ${ }^{8,39}$

In antibiotic-producing Streptomyces strains, it has been demonstrated that both morphological differentiation and secondary metabolism are controlled by a microbial hormone, $\gamma$-butyrolactone, similar to the A-factor (2-isocaryloyl $3 R$-hydroxymethyl - $\gamma$-butyrolactone) found in the Sm producer. The factor accumulates gradually in a growth-dependent manner by the action of AfsA. ${ }^{41}$ When the A-factor 
concentration reaches a critical level, it binds to the A-factor-specific receptor (ArpA) that is bound to the adpA promoter and dissociates ArpA from the promoter, resulting in the induction of adpA transcription. ${ }^{42}$ AdpA activates the transcription of many genes for morphological differentiation and secondary metabolism, including the expression of Sm 6-phosphotransferase. ${ }^{43}$

Our group has also investigated self-resistance mechanisms on puromycin-producing Streptomyces alboniger, streptothricin-producing Streptomyces sp. V-13-1 and blasticidin S-producing Streptomyces morookaensis and reported on it in a review article. ${ }^{44}$ Although these three producers have ribosomes susceptible to each antibiotic's own product, they maintain self-resistance by producing puromycin-, streptothricin- or blasticidin S acetyltransferase, respectively, to inactivate their own product. Interestingly, aminoglycoside adenyltransferase produced by some bacteria resistant to aminoglycoside antibiotics has not yet been detected in the antibiotic-producing Streptomyces strains. The existences of antibiotic-producing microorganisms having ribosomes tolerant to their own antibiotic product were shown by other research groups. ${ }^{45-47}$

\section{ANTIBIOTICS THAT INHIBIT BACTERIAL CELL WALL SYNTHESIS}

D-Cycloserine (D-4-amino-3-isoxazolidone: D-CS), which is produced by Streptomyces. garyphalus and S. lavendulae, is a cyclic structural analog of D-alanine (D-Ala). Although the compound is an effective antimycobacterial agent, it is used only in combined therapies due to its serious side effects. ${ }^{48}$ Side effects are caused by the binding of D-CS to $N$-methyl-D-aspartate receptors as an agonist. However, application of these adverse effects to treatments for neural diseases ${ }^{49}$ such as Alzheimer's disease ${ }^{50}$ and Parkinsonism ${ }^{51}$ has been researched with great dedication.

The peptidoglycan layer composed of bacterial cell walls is the main component enabling bacteria to be resistant to osmotic pressure. The formation of UDP- $N$-acetyl muramyl pentapeptide, which is a precursor of peptidoglycan, is followed by a cross-linked reaction of the precursors. In the cross-linking process, D-Ala has an important role as a bridge molecule. ${ }^{52}$ As D-amino acids are not primarily found in natural resources, bacteria generate D-Ala from L-Ala by the catalytic activity of Ala racemase (ALR). This enzyme needs a pyridoxal 5'-phosphate (PLP) as a cofactor to catalyze the racemization of both Ala enantiomers. E. coli and Salmonella enterica serovar Typhimurium produce two kinds of closely related ALR-encoded genes (alr and dadX in E. coli, and dal and dadB in Salmonella typhimurium) ${ }^{53-55}$ For example, the racemase encoded by dal from $S$. enterica serovar Typhimurium is necessary for peptidoglycan synthesis and displays a $40 \%$ identity to a catabolic racemase encoded by $d a d B .^{56}$

$\mathrm{D}$-Ala is a substrate to form D-alanyl-D-alanine (D-Ala-D-Ala). ${ }^{57}$ The dipeptide is formed by the action of an ATP-dependent enzyme and a D-Ala-D-Ala ligase (DDL) and is incorporated into the PG precursor by the catalytic activity of the D-Ala-D-Ala-adding enzyme. ${ }^{52}$ E. coli produces two kinds of DDL, designated DdlA and DdlB, which are encoded by $d d l A$ and $d d l B$, respectively. S. enterica serovar Typhimurium expresses DDL, which has a high similarity to the E. coli DdlA. ${ }^{58}$

D-CS interferes with the activities of both ALR and DDL, which are necessary for the synthesis of PG contained in the cell walls of bacteria. As these enzymes are unique to bacteria, they may become potential targets for the screening of selective antibacterial agents. ${ }^{59}$ ALR and DDL have been considered to be competitively inhibited, because D-CS is structurally similar to D-Ala. ${ }^{58,60}$ However, it has been recently reported that D-CS inhibits the catalytic activity of ALR via a time-dependent inactivation manner. ${ }^{58}$ In addition, the antibiotic and its enantiomer, L-cycloserine, inhibit several kinds of PLPdependent enzymes in the same manner. ${ }^{61-63}$

We have first cloned a 3.5-kb DNA fragment that carries a gene, which confers resistance to D-CS from D-CS-producing S. garyphalus. ${ }^{64}$ The hydropathy plot analysis of a protein deduced from the nucleotide sequence of the gene that encodes D-CS resistance revealed that the protein may carry membrane-integral domains that span the membrane 10 times, suggesting that the D-CS resistance gene product may be a factor associated with D-CS transport. An incomplete gene was found to be located upstream of the transmembrane protein gene from S. garyphalus. The incomplete gene consists of $246 \mathrm{bp}$ and the putative protein has a $52.6 \%$ identity with a DDL from $P$. aeruginosa. ${ }^{65}$ On the other hand, although the cloned fragment has a few ORFs, no ORF is similar to a gene that encodes ALR. As DDL and ALR are target enzymes of D-CS, it is of great interest to know whether these enzymes from the D-CS-producing microorganism show resistance to D-CS.

We have also cloned ALR- and DDL-encoding genes from another D-CS-producing S. lavendulae, ATCC25233. ${ }^{66}$ Both of the S. lavendulae ALR and DDL, which were overproduced in an E. coli host-vector system, were purified and characterized biochemically and kinetically. When compared with the $K_{\mathrm{i}}$ values for the E. coli DdlA and DdlB, that for the S. lavendulae DDL $(920 \mu \mathrm{M})$ was obviously higher than that for DdlA $(9 \mu \mathrm{M})$ and DdlB $(27 \mu \mathrm{M}){ }^{66}$ We have also shown that the catalytic activity of ALR from D-CS-producing S. lavendulae is scarcely inhibited by D-CS. ${ }^{6}$ Thus, the kinetic study for ALA and DDL has demonstrated that both enzymes from D-CS-producing S. lavendulae function as the self-resistance determinants.

To obtain structural evidence for the fact that the Streptomyces ALR is resistant to D-CS, we have determined the tertiary structures of the D-CS-free and D-CS enantiomer-bound forms of the Streptomyces ALR at high resolutions. ${ }^{67}$ The Streptomyces ALR takes a dimer structure, which is formed by interactions between the N-terminal domain of one monomer with the C-terminal domain of its partner

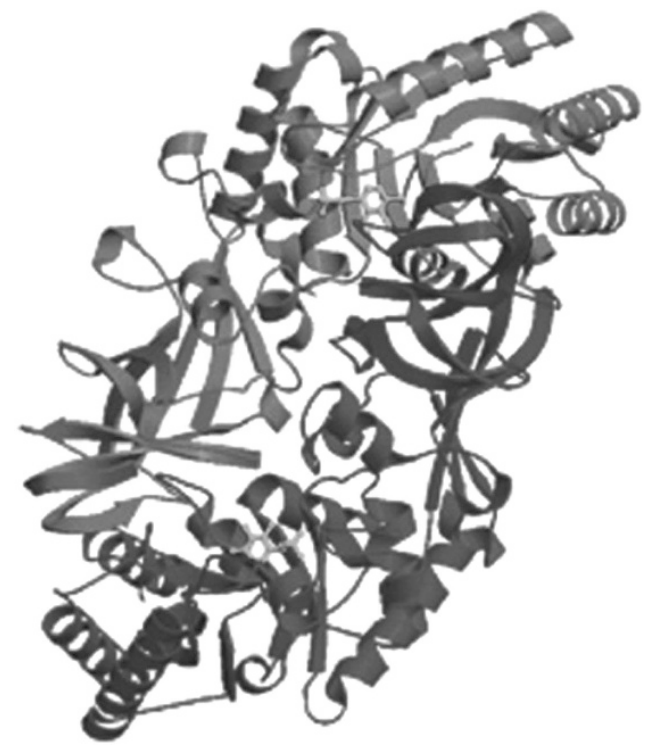

Figure 6 Overall structure of alanine racemase from D-cycloserine-producing S. lavendulae. A full color version of this figure is available at The Journal of Antibiotics journal online. 
(Figure 6). Each of the two active sites of ALR, which is generated as a result of the formation of the dimer structure, is composed of PLP, the PLP-binding residue Lys38 and the amino acids in the immediate environment of the pyridoxal cofactor. The current model suggests that each active site of Streptomyces ALR maintains a larger space and takes a more rigid conformation than that of the Bacillus stearothermophilus ALR determined previously by another group. Furthermore, we have shown that the addition of each isomer of cycloserine to Streptomyces ALR results in a slow conversion to a final form of a pyridoxal derivative arising from either isomer of cycloserine, which inhibits the catalytic activity noncompetitively. In fact, the slow conversion is confirmed by the fact that each enzyme-bound PLP derivative that is bound to PLP takes a different structure at the two active sites in the dimer.

We have successfully cloned the D-CS biosynthetic gene cluster from a D-CS-producing S. lavendulae ATCC $11924 .{ }^{68}$ The putative gene cluster consists of $10 \mathrm{ORFs}$, designated $d c s A$ to $d c s J$. This cluster includes two genes that encode DDL $(d c s I)$ and a putative membrane protein $(d c s J)$, which are the self-resistance determinants of the D-CS producer. Thus, to avoid self-inhibition by own antibiotic product, the producer organisms often encode cognate export and/or self-resistance mechanisms in the biosynthetic gene clusters.

\section{CONFLICT OF INTEREST}

The author declares no conflict of interest

\section{ACKNOWLEDGEMENTS}

I thank Drs T Kumagai, Y Matoba, H Matsuo and M Noda, who are brilliant staff members in my laboratory. I also thank the Emeritus Professors R Nomi of Hiroshima University and Julian E Davies of Institute Pasteur Paris, who enlightened me to this research. I am grateful to the beamline staff at SPring-8, Japan, for their kind help with X-ray data collection and thank the Japan Aerospace Exploration Agency (JAXA) for the crystallographic experiment.

1 Goodfellow, M. Ecology of actinomycetes. Ann. Rev. Microbiol. 37, 189-216 (1983).

2 Magnet, S. \& Blanchard, J. Molecular insights into aminoglycoside action and resistance. Chem. Rev. 105, 477-497 (2005).

3 Friedland, I. R. \& McCracken, G. H. Jr. Management of infections caused by antibioticresistant Streptococcus pneumoniae. N. Engl. J. Med. 331, 377-382 (1994).

4 Koornhof, K. J., Wasas, A. \& Klungman., K. Antimicrobial resistance in Streptococcus pneumoniae: a South African perspective. Clin. Infec. Dis. 15, 84-94 (1992).

5 Plorde, J. J. \& Sherris, J. C. Staphylococcal resistance to antibiotics: origin, measurement, and epidemiology. Ann. N. Y. Acad. Sci. 236, 413-434 (1974).

6 Pokkunuri, I. \& Champney, W. Characteristics of a 50 S ribosomal subunit precursor particle as a substrate for ermE methyltransferase activity and erythromycin binding in Staphylococcus aureus. RNA Biol. 4, 147-153 (2007)

7 Berens, C. \& Hillen, W. Gene regulation by tetracyclines. Constraints of resistance regulation in bacteria shape TetR for application in eukaryotes. Eur. J. Biochem. 270, 3109-3121 (2003).

8 Sugiyama, M. \& Nimi, O. Streptomycin biosynthesis and self-resistance mechanism in Streptomycin-producing Streptomyces griseus. Actinomycetol 4, 15-22 (1990).

9 Umezawa, H., Maeda., K., Takeuchi, T. \& Okami, Y. New antibiotics, bleomycin A and B. J. Antibiot. 19, 200-209 (1966).

10 Blum, R. H., Carter, S. K. \& Agre, K. A clinical review of bleomycin - a new antineoplastic agent. Cancer 31, 903-914 (1973).

11 Umezawa, H. Chemistry and mechanism of action of bleomycin. Fed. Proc. 33, 2296-2302 (1974).

12 Stubbe, J. \& Kozarich, J. W. Mechanism of bleomycin-induced DNA degradation. Chem. Rev. 87, 1107-1136 (1987).

13 Burger, R. M., Peisach, J. \& Horwits, S. B. Activated bleomycin. J. Biol. Chem. 256, 11636-11644 (1981).

14 Sugiyama, M. et al. Charaterisation by molecular cloning of two genes from Streptomyces verticillus encoding resistance to bleomycin. Gene 151, 11-16 (1994).

15 Sugiyama, M., Kumagai., T., Shionoya, M., Kimura, E. \& Davies, J. E. Inactivation of bleomycin by an $N$-acetyltransferase in the bleomycin-producing strain Streptomyces verticillus. FEMS Microbiol. Lett. 121, 81-86 (1994).
16 Drocourt, D., Calmels, T., Reynes, J.-P., Baron, M. \& Tiraby, G. Cassettes of the Streptoalloteichus hindustanus ble gene for transformation of lower and higher eukaryotes to phleomycin resistance. Nucleic Acids Res. 18, 4009 (1990)

17 Kumagai, T. et al. Crystallization and preliminary X-ray diffraction studies of bleomycinbinding protein from bleomycin-producing Streptomyces verticillus. Acta Crystallogr. D 54, 127-128 (1998).

18 Kawano, Y. et al. The $1.5 \AA$ crystal structure of a bleomycin resistance determinant from bleomycin-producing Streptomyces verticillus. J. Mol. Biol. 295, 915-925 (2000).

19 Sugiyama, M. \& Kumagai, T. Molecular and structural biology of bleomycin and its resistance determinants. J. Biosci. Bioeng. 93, 105-116 (2002).

20 Sugiyama, M., Kumagai, T., Hayashida, M., Maruyama, M. \& Matoba, Y. The 1.6- $\AA$ crystal structure of the copper(II)-bound bleomycin complexed with the bleomycinbinding protein from bleomycin-producing Streptomyces verticillus. J. Biol. Chem. 277, 2311-2320 (2002).

21 Berg, D. E., Davies, J., Allet, B. \& Rochaix, J. D. Transposon of R factor genes to bacteriophage lambda. Proc. Natl Acad. Sci. USA 72, 3628-3632 (1975).

22 Genilloud, O., Garrido, M. C. \& Moreno, F. The transposon Tn5 carries a bleomycinresistance determinant. Gene 32, 225-233 (1984).

23 Mazodier, P., Cossart, P., Giraud, E. \& Gasser, F. Completion of the nucleotide sequence of the central region Tn5 confirms the presence of three resistance genes. Nucleic Acids Res. 13, 185 (1985).

24 Kumagai, T., Maruyama, M., Matoba, Y., Kawano, Y. \& Sugiyama, M. Crystallization and preliminary $\mathrm{X}$-ray diffraction studies of bleomycin-binding protein encoded on the transposon Tn5. Acta Crystallogr. D 55, 1095-1097 (1999).

25 Maruyama, M. et al. Crystal structures of the transposon Tn5-carried bleomycin resistance determinant uncomplexed and complexed with bleomycin. J. Biol. Chem. 276, 9992-9999 (2001)

26 Rubin, S. J. \& Rosenblum, E. D. Effects of ethidium bromide on growth and on loss of the penicillinase plasmid of Staphylococcus aureus. J. Bacteriol. 108, 1200-1204 (1971).

27 Bhuiyan, M. Z., Ueda, K., Inouye, Y. \& Sugiyama, M. Molecular cloning and expression in Escherichia coli of bleomycin-resistance gene from a methicillin-resistant Staphylococcus aureus and its association with IS431mec. Appl. Microbiol. Biotechnol. 43, 65-69 (1995).

28 Sugiyama, M. et al. IS431mec-mediated integration of a bleomycin-resistance gene into the chromosome of a methicillin-resistant Staphylococcus aureus strain isolated in Japan. Appl. Microbiol. Biotechnol. 46, 61-66 (1996).

29 McElgunn, C. J., Bhuyian, M. Z. A. \& Sugiyama, M. Integration analysis of pSK41 in the chromosome of a methicillin-resistant Staphylococcus aureus K-1. J. Basic Microbiol. 42, 190-200 (2002)

30 Kumagai, T., Nakano, T., Maruyama, M., Mochizuki, H. \& Sugiyama, M. Characterization of the bleomycin resistance determinant encoded on the transposon Tn5. FEBS Lett. 442, 34-38 (1999).

31 Oda, K., Matoba, Y., Noda, M., Kumagai, T. \& Sugiyama, M. Catalytic mechanism of bleomycin $\mathrm{N}$-acetyltransferase proposed on the basis of its crystal structure. J. Biol. Chem. 285, 1446-1456 (2010).

32 Danshiitsoodol, N., de Pinho, C. A., Matoba, Y., Kumagai, T. \& Sugiyama, M. The mitomycin C (MMC)-binding protein from MMC-producing microorganisms protects from the lethal effect of bleomycin: crystallographic analysis to elucidate the binding mode of the antibiotic to the protein. J. Mol. Biol. 360, 398-408 (2006).

33 Sheldon, P. J., Johnson, D. A., August, P. R., Liu, H. W. \& Sherman, D. H. Characterization of a mitomycin-binding drug resistance mechanism from the producing organism Streptomyces lavendulae. J. Bacteriol. 179, 1797-1804 (1997).

$34 \mathrm{He}$, M., Sheldon, P. J. \& Sherman, D. H. Characterization of a quinine reductase activity for the mitomycin $\mathrm{C}$ binding protein (MRD): Functional switching from a drug-activating enzyme to a drug-binding protein. Proc. Natl Acad. Sci. USA 98, 926-931 (2001).

35 Martin, T. W. et al. Molecular basis of mitomycin C resistance in Streptomyces: structure and function of the MRD protein. Structure 10, 933-942 (2002).

36 Rife, C. L., Pharris, R. E., Newcomer, M. E. \& Armstrong, R. N. Crystal structure of a genomically encoded fosfomycin resistance protein (FosA) at $1.9 \AA$ resolution by MAD phasing off the L-IIl edge of TI+. J. Am. Chem. Soc. 124, 11001-11003 (2002).

$37 \mathrm{He}$, M. M., Clugston, S. L., Honek, J. F. \& Mathews, B. W. Determination of the structure of Escherichia coliglyoxylase I suggests a structural basis for differential metal activation. Biochemistry 39, 8719-8728 (2000).

38 WHO Anti-tuberculosis drug resistance in the world, Report No. 3 (2004).

39 Sugiyama, M., Mochizuki, H., Nimi, O. \& Nomi, R. Mechanism of protection of protein synthesis against streptomycin inhibition in a producing strain. J. Antibiot. 34, 1183-1188 (1981).

40 Sugiyama, M., Kobayashi, H., Nimi, O. \& Nomi, R. Susceptibility of protein synthesis to streptomycin in streptomycin-producing Streptomyces griseus. FEBS Lett. 110, 250-252 (1980)

41 Kato, J., Funa, N., Watanabe, H., Ohnishi, Y. \& Horinouchi, S. Biosynthesis of $\gamma$-butyrolactone autoregulators that switch on secondary metabolism and morphological development in Streptomyces. Proc. Natl Acad. Sci. USA 104, 2378-2383 (2007).

42 Onishi, Y., Kameyama, S., Onaka, H. \& Horinouchi, S. The A-factor regulatory cascade leading to streptomycin biosynthesis in Streptomyces griseus: identification of a target gene of the A-factor receptor. Mol. Microbiol. 34, 102-111 (1999).

43 Hara, H., Onishi, Y. \& Horinouchi, S. DNA microarray analysis of global gene regulation by A-factor in Streptomyces griseus. Microbiology 155, 2197-2210 (2009).

44 Sugiyama, M., Kumagai, T. \& Matoba, Y. [Self-protection mechanisms of antibioticproducing microorganisms]. Nihon Rinsho 65, 669-673 (2007). 
45 Thompson, J., Schmidt, F. \& Cundliffe, E. Site of action of a ribosomal RNA methylase conferring resistance to thiostrepton. J. Biol. Chem. 257, 7915-7917 (1982).

46 Bechthold, A. \& Floss, H. G. Overproduction of the thiostrepton-resistance gene from Streptomyces azureus in Escherichia coli and characterization of recognition sites of the $23 \mathrm{~S}$ rRNA A1067 2'-metyltransferase in the guanosine triphosphatase center of 23S ribosomal RNA. Eur. J. Biochem. 224, 431-437 (1994).

47 Hotta, K., Ishikawa, J., Ogata, T. \& Mizuno, S. Secondary aminoglycoside resistance in aminoglycoside-producing strains of Streptomyces. Gene 115, 113-117 (1992).

48 Heifets, L. B. \& Iseman, M. D. Individualized therapy versus standard regimens in the treatment of Mycobacterium avium infections. Am. Rev. Respir. Dis. 144 $1-2$ (1991)

49 Johannesen, T. S. \& Myhrer, T. Impaired visual memory in rats reared in isolation is reversed by D-cycloserine in the adult rat. Eur. J. Pharmacol. 437, 73-77 (2002)

50 Andersen, J. M., Lindberg, V. \& Myhrer, T. Effects of scopolamine and D-cycloserine on non-spatial reference memory in rats. Behav. Brain Res. 129, 211-216 (2002).

51 Schneider, J. S., Tinker, J. P., Van Velson, M. \& Giardiniere, M. Effects of the partial glycine agonist D-cycloserine on cognitive functioning in chronic low dose MPTP-treated monkeys. Brain Res. 860, 190-194 (2000).

52 Walsh, C. T. Enzymes in the D-alanine branch of bacterial cell wall peptidoglycan assembly. J. Biol. Chem. 264, 2393-2396 (1989).

53 Wasserman, S. A., Daub, E., Grisafi, P., Botstein, D. \& Walsh, C. T. Catabolic alanine racemase from Salmonella typhimurium: DNA sequence, enzyme purification, and characterization. Biochemistry 23, 5182-5187 (1984).

54 Esaki, N. \& Walsh, C. T. Biosynthetic alanine racemase of Salmonella typhimurium: purification and characterization of the enzyme encoded by the alr gene. Biochemistry 25, 3261-3267 (1986)

55 Lilley, P. E., Stamford, N. P., Vasudevan, S. G. \& Dixon, N. E. The 92-min region of the Escherichia coli chromosome: location and cloning of the ubiA and alr genes. Gene 129, 9-16 (1993).

56 Galakatos, N. G., Daub, E., Botstein, D. \& Walsh, C. T. Biosynthetic alr alanine racemase from Salmonella typhimurium: DNA and protein sequence determination. Biochemistry 25, 3255-3260 (1986).
57 Neuhaus, F. C. The enzymatic synthesis of D-alanyl-D-alanine. I. Purification and properties of D-alanyl-D-alanine synthetase. J. Biol. Chem. 237, 778-786 (1962).

58 Zawadzke, L. E., Bugg, T. D. H. \& Walsh, C. T. Existence of two D-alanine:D-alanine ligases in Escherichia coli: cloning and sequencing of the ddIA gene and purification and characterization of the DdlA and DdlB enzymes. Biochemistry 30 1673-1682 (1991).

59 Neuhaus, F. C. \& Hammes, W. P. Inhibition of cell wall biosynthesis by analogues and alanine. Pharmacol. Ther. 14, 265-319 (1981).

60 Lambert, M. P. \& Neuhaus, F. C. Mechanism of D-cycloserine action: alanine racemase from Escherichia coli W. J. Bacteriol. 110, 978-987 (1972).

61 Fenn, T. D., Stamper, G. F., Morollo, A. A. \& Ringe, D. A side reaction of alanine racemase: transamination of cycloserine. Biochemistry 42, 5775-5783 (2003).

62 Peisach, D., Chipman, D. M., van Ophem, P. W., Manning, J. M. \& Ringe, D. Crystallographic study of steps along the reaction pathway of D-amino acid aminotransferase. Biochemistry 37, 4958-4967 (1998).

63 Wang, E. \& Walsh, C. T. Suicide substrates for the alanine racemase of Escherichia coli B. Biochemistry 17, 1313-1321 (1978).

64 Matsuo, H., Kumagai, T., Mori, K. \& Sugiyama, M. Molecular cloning of a D-cycloserine resistance gene from D-cycloserine-producing Streptomyces garyphalus. J. Antibiot. 56 762-767 (2003).

65 Stover, C. K. et al. Complete genome sequence of Pseudomonas aeruginosa PAO1, an opportunistic pathogen. Nature 406, 959-964 (2000).

66 Noda, M. et al. Self-protection mechanism in D-cycloserine-producing Streptomyces lavendulae: gene cloning, characterization, and kinetics of its alanine racemase and D-alanyl-alanine ligase which are target enzymes of D-cycloserine. J. Biol. Chem. 279 46143-46152 (2004).

67 Noda, M., Matoba, Y., Kumagai, T. \& Sugiyama, M. Structural evidence that alanine racemase from $\mathrm{D}$-cycloserine-producing microorganism exhibits resistance to its own product. J. Biol. Chem. 279, 46153-46161 (2004).

68 Kumagai, T. et al. Molecular cloning and heterologous expression of a biosynthetic gene cluster for the antitubercular agent D-cycloserine produced by Streptomyces lavendulae. Antimicrob. Agents Chemother. 54, 1132-1139 (2010). 